\title{
The use of gadolinium-containing medium dilutions in evaluations of pathological changes in magnetic resonance images of the canine elbow
}

\author{
Yauheni Zhalniarovich, Paulina Przyborowska-Zhalniarovich, \\ Joanna Głodek, Zbigniew Adamiak
}

University of Warmia and Mazury in Olsztyn, Department of Surgery and Radiology, Olsztyn, Poland

Received May 23, 2017

Accepted August 13, 2018

\begin{abstract}
The aim of this study was to evaluate the usefulness of the paramagnetic gadoliniumcontaining contrast agent, diluted 1:800, in evaluations of pathological changes in the canine elbow joint. The experiment was performed on 6 large breed dogs of both sexes with a body weight of 25 to $40 \mathrm{~kg}$. Thoracic limb lameness and pain in the elbow joint area were observed in all patients. The animals were subjected to standard physical examinations, radiography and low-field magnetic resonance imaging scans with the use of a contrast agent. The Spin Echo T1 dorsal sequence as well as 3D SST1 transverse and XBONE T1 transverse sequences were highly effective in diagnosing osteochondritis dissecans (OCD) of the medial humeral condyle. Degenerative changes and the fragmented coronoid process (FCP) of the ulna were very well visualized by High Resolution Gradient Echo, XBONE T2 and Spin Echo T1 sequences in the sagittal plane. The administration of the gadolinium contrast agent, diluted 1:800, to the elbow joint cavity enhances the diagnostic value of magnetic resonance images in evaluations of medial compartment disease, in particular fragmentation of the medial coronoid process.
\end{abstract}

MRI, arthrography, contrast agent, dogs, elbow

Thoracic limb lameness caused by pathological changes in the elbow joint is difficult to diagnose (Reichle and Snaps 1999). Magnetic resonance imaging (MRI) is a noninvasive and highly sensitive tool for evaluating soft tissues of the musculoskeletal system (Miller 1999; Snaps et al. 1999). This method is routinely used to diagnose pathological changes in the medial epicondyle in humans, and it is recommended when other techniques, such as radiography, ultrasonography and clinical examinations, fail to provide a clear diagnosis (Park et al. 2008). In human medicine, MRI is a method of choice for diagnosing lesions and diseases of the elbow joint and other joints with cavities (Dalinka et al. 1989; De Smet et al. 1990; Hayes and Conway 1992; Heron and Calvert 1992; Hodler et al. 1992; Recht 1994; Buckwalter 1996; Hill et al. 2000; Sahim and Demirtas 2006). According to Cook and Cook (2009), MR images are a source of additional information for diagnosing pathological changes in tendons and muscles in the area of the canine elbow joint. Canine elbow dysplasia was long diagnosed by radiography and arthroscopy. Today, older diagnostic methods are being gradually replaced by MRI (Adamiak et al. 2011). The standard MRI protocol for examining the elbow joint involves T1- and T2-weighted sequences in dorsal, sagittal and transverse planes (Adamiak et al. 2011; Zhalniarovich et al. 2014; Zhalniarovich et al. 2017).

The main aim of this study was to determine the diagnostic usefulness of the paramagnetic gadolinium-containing contrast medium, diluted 1:800 and administered to the elbow joint.

\section{Materials and Methods}

All patients included in this study were referred for assessment of unilateral forelimb lameness. The study was performed on 6 elbow joints of large breed dogs (6 client-owned dogs) of both sexes, including 3 German

Address for correspondence:

Y. Zhalniarovich

Department of Surgery and Radiology

University of Warmia and Mazury in Olsztyn

Oczapowskiego 14, 10-957 Olsztyn, Poland

Phone: +48895233795

E-mail: eugeniusz.zolnierowicz@uwm.edu.pl

http://actavet.vfu.cz/ 
Shepherds, 2 Labrador Retrievers and 1 mixed-breed. The mean age was 3.3 years ( 7 months to 6 years), with a body weight of 25 to $40 \mathrm{~kg}$, showing lameness in the thoracic limb. Clinical and orthopaedic examinations revealed that pain was localized in the area of the elbow joint. All animals were subjected to complete orthopaedic examinations; lateral, flexed lateral, and craniocaudal radiographic projections; and pre- and post-contrast MRI of the elbow joint. The contrast agent was gadolinium-containing medium (Omniscan, GE Healthcare, Princeton NJ, USA, $0.5 \mathrm{mmol} / \mathrm{ml}$ ), diluted 1:800 and administered to the elbow joint cavity. Preliminary studies had revealed that the 1:800 ratio was the optimal dilution for MRI of the elbow joint (Zhalniarovich et al. 2017). Gadolinium was diluted 1:800 with the use of $0.01 \mathrm{ml}(10 \mu \mathrm{l})$ of the Omniscan contrast agent and $8 \mathrm{ml}$ of water for injection (Aqua pro injectione, Polpharma, Starogard Gdański, Poland). The contrast agent was administered to the elbow joint at the amount of 1-2 $\mathrm{ml}$, and it was distributed evenly by flexing and extending the joint.

The patients were examined in a low-field $(0.25 \mathrm{~T}) \mathrm{MRI}$ scanner with a permanent magnet (Vet-MR Grande, Esaote, Genova, Italy) with the involvement of the ankle/foot DPA coil used in human medicine. For radiographic examination all the animals were premedicated. After radiographic modality all the patients were positioned inside the MRI coil. The elbow joint was scanned in three planes: dorsal, sagittal and transverse. Each patient was examined with the use of identical MRI sequences: Spin Echo T1 in the dorsal plane; High Resolution Gradient Echo, Spin Echo T1, and XBONE T2 in the sagittal plane; and XBONE T1 and 3D SST1 in the transverse plane. The scanning time was 35-40 min. The contrast agent was administered to the elbow joint cavity, and the above MRI sequences were repeated in every patient to determine the contrast agent's influence on the diagnostic quality of the resulting MR images. The obtained MR images were evaluated for intensity of surrounding elbow joint tissues, joint space brightness, homogeneous consistency and intensity of the contrast medium. All obtained images were subjectively interpreted by all co-authors (four people) of the publication blindly to each other.

\section{Results}

For all six joints and MRI sequences, the signal intensity was assessed for joint capsule, bone marrow, subchondral bone, articular cartilage, ligaments and contrast medium. Two dogs $(33.3 \%)$ were diagnosed with osteochondritis dissecans (OCD) of the medial humeral condyle. Four dogs (66.6\%) had fragmented coronoid process (FCP) of the ulna. In Spin Echo T1 and XBONE T2 sequences, there was a homogenous iso- to low-intensity signal of the subchondral bone and FCP compared to the surrounding muscles. The results of the study indicate that the Spin Echo T1 sequence in the dorsal plane was highly useful for diagnosing OCD of the medial humeral condyle. Degenerative changes in the medial condyle were also visualized in 3D SST1 and XBONE T1 sequences in the transverse plane (Plate IV, Fig. 1).

On the sagittal plane, the medial coronoid process and the medial aspect of the humeral condyle were clearly illustrated. On these planes the trochlear notch of the ulna and the anconeal process appeared smooth and curved. In sagittal and dorsal planes two opposing articular cartilages could not be seen separately. Our findings suggest that High Resolution Gradient Echo, XBONE T2 and Spin Echo T1 sequences in the sagittal plane are very useful for diagnosing degenerative changes and fragmentation of the coronoid process of the ulna (Plate IV, Fig. 2a). The joint capsule and ligaments had low signal intensity on all MRI sequences. The contacted cartilage surfaces were difficult to differentiate on all sequences.

On post-contrast MR images, the joint cavity was hyperintense compared to the signal for surrounding muscles. For 4 joints, a clearly hyperintense signal received from the gadolinium contrast agent was seen between the FCP and ulna (Fig. 2b).

The results of MRI scans revealed that the lameness was caused by degenerative changes of the medial humeral condyle of the elbow in two dogs and by FCP in the remaining four patients.

\section{Discussion}

The canine elbow is a hinge joint with an articular capsule that constitutes the articular cavity. In humans, MRI is used to detect loose cartilage bodies in joints and collateral ligaments of joints. Magnetic resonance exams can be performed with the use of a contrast agent to enhance the diagnostic quality of the test (Hill et al. 2000). 
In veterinary medicine, MRI is still relatively rarely used in examinations of the elbow joint, and the literature on the subject is largely limited (Van Bree and Gielen 2008; Baeumlin et al. 2009; Cook and Cook 2009).

Our study indicates that the Spin Echo T1 sequence in the sagittal plane produces elbow joint images of low diagnostic value. The above sequence is also characterized by a short scan time. According to Baeumlin et al. (2009), the anatomy of the elbow joint can be visualized with high resolution when T1-weighted sequences are used. T2-weighted sequences produce less detailed images of elbow anatomy, but they effectively visualize cartilage defects. Our experiment suggests that XBONE T2 and High Resolution Gradient Echo sequences in the sagittal plane produce high-resolution images and support diagnoses of the fragmented medial coronoid process of the ulna. XBONE T1 and 3D SST1 sequences in the transverse plane can be effectively deployed to evaluate the articular cavity, surfaces of the elbow joint and osteochondritis dissecans of the medial humeral condyle. In Spin Echo T1 and XBONE T2 sequences, there was iso- to low-intensity signal of the subchondral bone and fragmented coronoid process compared to the surrounding muscles. Our results corroborate the findings of Baeumlin et al. (2009) who noted that MR examinations of the elbow joint also support detailed evaluations of the surrounding structures.

Dysplastic changes in elbow joints have not yet been fully explored (Cook and Cook 2009), which is why the term "medial compartment disease" is increasingly often used to replace "fragmentation of the medial coronoid process" (Gemmill 2013). As demonstrated in our study, osteochondritis dissecans is most effectively diagnosed in sagittal and dorsal planes in the form of loss of shadowing, flattened or irregular surfaces, or erosion of the medial condyle. Defects can reach the osseous layer of the condyle. According to Reichle and Snaps (1999) and Probst et al. (2007), MRI sequences in sagittal and dorsal planes are highly effective in diagnosing osteochondritis dissecans of the medial humeral condyle, but deliver far less detailed images of minor cartilage defects that do not reach subcartilaginous tissue. The above limitation can be attributed to the fact that cartilage is very thin in the discussed area. Soft tissue mineralization at the level of the medial condyle of the humerus can also point to dysplastic changes in the elbow joint (Grondalen and Braut 1976; Zontine et al. 1989; Walker 1998; Harasen 2003).

The results of an MRI examination of ununited anconeal process of the canine ulna were described in only one research study. The change was visualized in the sagittal plane, and it consisted of a groove between the anconeal process and the ulna (Reichle and Snaps 1999).

According to Carpenter et al. (1993) and Snaps et al. (1997), the detectability of pathological changes in the medial condyle of the humerus was estimated at $77 \%$ for MRI and $72 \%$ for radiography, whereas the detectability of the fragmented medial coronoid process was determined at $95.5 \%$ for MRI, $77 \%$ for radiography, and $86.7 \%$ for computed tomography. The above results indicate that MR is the most effective imaging technique for diagnosing pathological changes in the medial compartment of elbow joint. The administration of the contrast agent to the elbow joint cavity improved the diagnostic accuracy of MRI images. In this report, a solution of $0.5 \mathrm{mmol} / \mathrm{ml}$ of gadolinium contrast was used to outline lesions of FCP. The 1:800 dilution of the contrast agent was selected as optimal on the basis of a previous report (Zhalniarovich et al. 2017). The contrast agent delivered superior resolution in evaluations of degenerative changes affecting the medial compartment of the elbow joint, in particular the fragmentation of the medial coronoid process (Plate IV, Fig. 2b). Magnetic resonance arthrography delineates lesions of the FCP. The enhancement of the gadolinium contrast was obtained by extending the space between the joint cavity and the bone defect. Magnetic resonance arthrography is more helpful when bone defects are involving the subchondral bone (Snaps et al. 1999). 
According to Beltran (1991) and Helgason (1997), the administration of the gadolinium contrast agent to joint cavities improves the visibility of pathological changes affecting the examined joints.

In our study, none of the patients experienced side effects resulting from the administration of the contrast agent. Hajek et al. (1990) concluded that gadolinium was a safe contrast agent for intra-articular injections administered to the joint cavity in rabbits.

Our report has some limitations. Firstly, the number of patients is small. Further studies on larger groups of dogs are needed to determine if the result remain the same. Another limitation is that the study involves only large breed dogs over $25 \mathrm{~kg}$. Additional research on small breed dogs is required.

It can be concluded that low-field MRI was found to be a highly sensitive technique for diagnosing dysplastic changes in the medial condyle of the humerus and fragmentation of the medial coronoid process of the ulna. Unlike radiographic tests which often produce false negative results, MRI supports the acquisition of high-quality images that are easier to interpret and facilitate the diagnosis of joint defects at early stages of disease (Reichle and Snaps 1999).

\section{References}

Adamiak Z, Jaskólska M, Matyjasik H, Pomianowski H, Kwiatkowska M 2011: Magnetic resonance imaging of selected limb joints in dogs. Pol J Vet Sci 14: 501-505

Baeumlin Y, De Rycke L, Van Caelenberg A, Van Bree H, Gielen I 2009: Magnetic resonance imaging of the canine elbow: an anatomic study. Vet Surg 39: 566-573

Beltran J 1991: Gadopentate dimeglumine-enhanced MR imaging of the musculoskeletal system. Am J Roentgenol 156: 457-466

Buckwalter KA 1996: Magnetic resonance of the knee. Radiologist 3: 99-122

Carpenter LG, Schwarz PD, Lowry JE, Park RD, Steyn PF 1993: Comparison of radiologic imaging techniques for diagnosis of fragmented medial coronoid process of the cubital joints in dogs. J Am Vet Med Assoc 203: 78-83

Cook CR, Cook JL 2009: Diagnostic imaging of canine elbow dysplasia: a review. Vet Surg 38: 144-153

Dalinka MK, Kricun ME, Zlatkin MB, Hibbard CA 1989: Modern diagnostic imaging in joint disease. Am J Roentgenol 152: 229-240

De Smet AA, Fisher DR, Graf BK, Lange RH 1990: Osteochondritis dissecans of the knee: value of MR imaging in determining lesion stability and the presence of cartilage defects. Am J Roentgenol 155: 549-553

Gemmill TJ 2013: Current understanding of medial compartment disease of the canine elbow. AOVET CourseSpecial focus in small animal fracture management, pp 30-34

Grondalen J, Braut T 1976: Lameness in two young dogs caused by calcified body in the joint capsule of the elbow. J Small Anim Pract 17: 681-684

Hajek PC, Sartoris DJ, Gylys-Morin V, Haghighi P, Engel A, Kramer F, Neumann CH, Resnick D 1990: The effect of intraarticular gadolinium-DTPA on synovial membrane and cartilage. Invest Radiol 25: 179-183

Harasen G 2003: The mysterious dysplastic elbow. Can Vet J 44: 673-674

Hayes CW, Conway FW 1992: Evaluation of articular cartilage: Radiographic and cross-sectional imaging techniques. Radiographics 12: 409-428

Helgason JW 1997: MR arthrography: a review of current technique and applications. Am J Roentgenol 168: 1473-1480

Heron CW, Calvert PT 1992: Three-dimensional gradient echo MR imaging of the knee: Comparison with arthroscopy in 100 patients. Radiology 183: 839-844

Hill NB, Bucchieri JS, Shon F, Miller TT, Rosenwasser MP 2000: Magnetic Resonance imaging of the injury to the medial collateral ligament of the elbow: a cadaver model. J Shoulder Elb Surg 9: 418-422

Hodler J, Berthiaume MJ, Scheitzer ME, Resnick D 1992: Knee joint hyaline cartilage defects: A comparative study of MR imaging and anatomic sections. J Comput Assist Tomo 16: 597-603

Miller TT 1999: Imaging of elbow disorders. Orthop Clin N Am 30: 21-36

Park GY, Lee SM, Lee MY 2008: Diagnostic value of ultrasonography for clinical medial epicondylitis. Arch Phys Med Rehab 89: 738-742

Probst A, Modler F, Kunzel W, Mlynarik V, Trattnig S 2007: Demonstration of the articular cartilage of the canine ulnar trochlear notch using high-field magnetic resonance imaging. Vet J 177: 63-70

Recht MP 1994: MR imaging of articular cartilage: current status and future directions. AJR Am J Roentgenol 24: $283-290$

Reichle JK, Snaps F 1999: The elbow. Clin Tech Small An P 14: 177-186 
Sahim G, Demirtas M 2006: An overview of MR arthrography with emphasis on the current technique and applicational hints and tips. Eur J Radiol 58: 416-430

Snaps FR, Balligand MH, Saunders JH, Park RD, Dondelinger RF 1997: Comparison of radiography, magnetic resonance imaging, and surgical findings in dogs with elbow dysplasia. Am J Vet Res 58: 1367-1370

Snaps FR, Park RD, Saunders JH, Balligand MH, Dondelinger RF 1999: Magnetic resonance arthrography of the cubital joint in dogs affected with fragmented medial coronoid processes. Am J Vet Res 60: 190-193

Van Bree H, Gielen I 2008: Diagnostic imaging in the elbow dysplasia: including scintigraphy, radiography, ultrasound, CT and MRI. Proceedings of $23^{\text {rd }}$ Annual Meeting International Elbow Working Group, Dublin, pp 13-17

Walker TM 1998: A redefined type of elbow dysplasia in the dog - 2 cases. Can Vet J 39: 573-575

Zhalniarovich Y, Adamiak Z, Glodek J, Przyborowska P, Holak P 2014: Comparison of High Resolution Gradient Echo, XBONE T1, XBONE T2, Spin Echo T1 and 3D SST1 magnetic resonance imaging sequences for imagining the canine elbow. Pol J Vet Sci 17: 587-591

Zhalniarovich Y, Przyborowska-Zhalniarovich P, Mieszkowska M, Adamiak Z 2017: Direct magnetic resonance arthrography of the canine elbow. Acta Vet Brno 86: 85-89

Zontine WJ, Weitcamp RA, Lippincott CL 1989: Redefined type of elbow dysplasia involving calcified flexor tendons attached to the medial humeral epicondyle in three dogs. J Am Vet Med Assoc 194: 1082-1085 


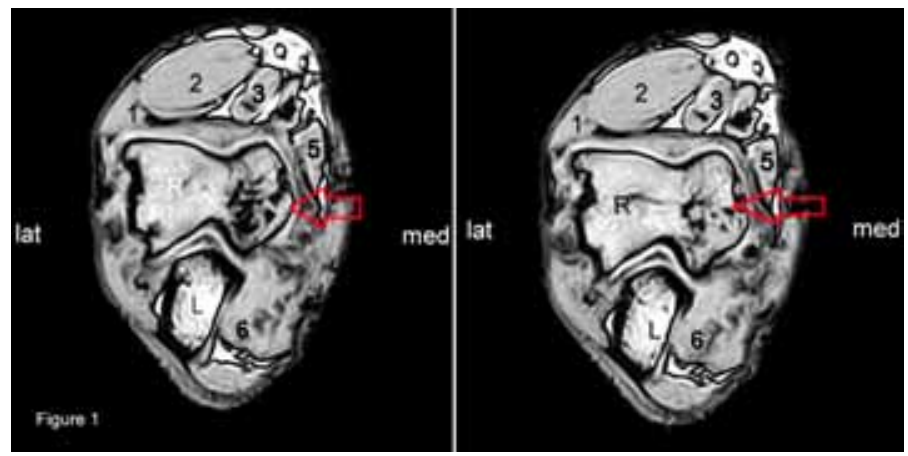

Fig. 1. Three-dimensional SST1 sequence in the transverse plane. Osteochondritis dissecans of the medial humeral condyle is marked with an arrow. Regenerating osseous tissue and osteophytes are visible on the medial side. $\mathrm{R}$ - humerus; L - ulna; 1 - extensor digitorum communis muscle; 2 - extensor carpi radialis misscle; 3 -brachialis muscle; 4 - biceps muscle; 5 - pronator teres muscle; 6 - flexor carpi ulnaris muscle.
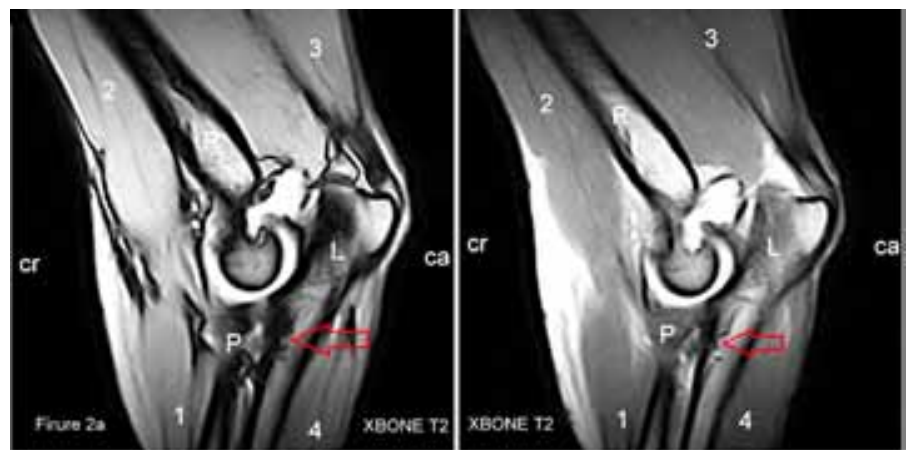

Fig. 2a. XBONE T2 sequence in the sagittal plane. Fragmented coronoid process (FCP) and the degenerative process in the elbow joint are marked with an arrow. $\mathrm{R}$ - humerus; $\mathrm{L}$ - ulna; $\mathrm{P}$ - radius; 1 - extensor carpi radialis muscle; 2 - biceps muscle; 3 - triceps muscle; 4 - flexor carpi ulnaris muscle.
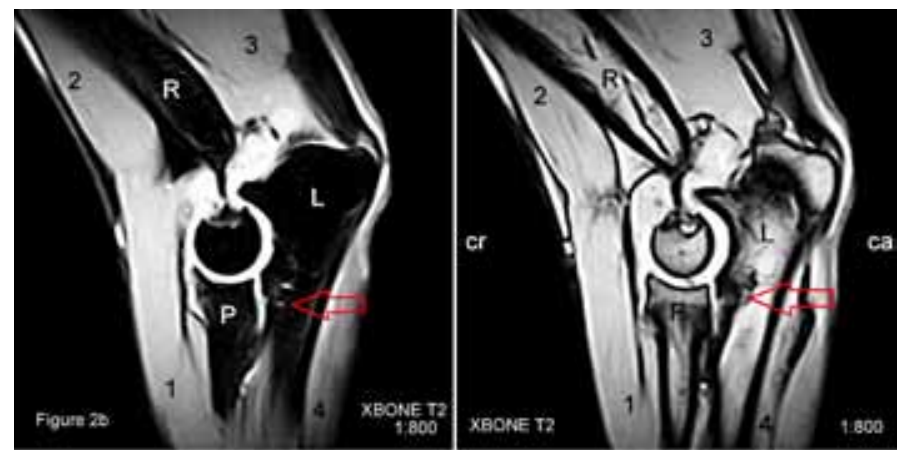

Fig. 2b. XBONE T2 sequence in the sagittal plane after the administration of the Omniscan contrast agent, diluted 1:800. Fragmented coronoid process (FCP) is marked with an arrow. The degenerative process in the elbow joint is visible. $\mathrm{R}$ - humerus; $\mathrm{L}$ - ulna; $\mathrm{P}$ - radius; 1 - extensor carpi radialis muscle; 2 - biceps muscle; 3 - triceps muscle; 4 - flexor carpi ulnaris muscle. 